\title{
ON COMMUTING TRANSFORMATIONS AND ROOTS ${ }^{1}$
}

\author{
J. R. BLUM AND N. FRIEDMAN
}

Let $(X, B, m)$ denote the measure space consisting of the unit interval with Lebesgue measure. We let $J$ denote the class of measurable nonsingular invertible transformations mapping $X$ onto $X$. Given $\tau \in J, \sigma$ commutes with $\tau$ if $\sigma(\tau(x))=\tau(\sigma(x))$ for a.e. $x \in X$ and $\sigma$ is a $p$ th root of $\tau$ if $\sigma^{p}(x)=\tau(x)$ for a.e. $x \in X$. If $\sigma$ is a root of $\tau$ then $\sigma$ commutes with $\tau$, hence it is of interest to find what properties of $\tau$ are inherited by $\sigma$ if $\sigma$ does commute with $\tau$ and in particular if $\sigma$ is a root of $\tau$. We consider some basic results along these lines in $\$ 1$. Concerning roots, it has been of interest to construct ergodic measure preserving transformations $\tau$ such that (1) $\tau$ has no roots of any order. More generally we consider (2) Let $P$ denote the primes $p \geqq 2$ and let $P=P_{1} \cup P_{2}$ where $P_{1}$ and $P_{2}$ are disjoint. $\tau$ has no $p$ th root for $p \in P_{1}$ but $\tau$ has a $p$ th root for $p \in P_{2}$. Ergodic measure preserving transformations with discrete spectrum satisfying (1) were constructed in [1]. In [4] ergodic measure preserving transformations with continuous spectrum satisfying (1) were constructed. We remark that the continuous spectrum case is quite difficult and it was only recently that transformations with continuous spectrum and no square root were constructed in [3]. In $\$ 2$ of this note we obtain ergodic measure preserving transformations with discrete spectrum satisfying (2). Our method depends on generalizing certain results in [6].

The question of whether every invertible mixing transformation has a square root is still open. In order to illuminate this problem, in $\$ 3$ we give some transparent examples of invertible mixing transformations with roots of all orders and also mention a class of mixing transformations for which square roots may not exist.

1. In what follows all transformations will be assumed to be in $\mathfrak{J}$ and to be ergodic. Our starting point is the following result.

THEOREM 1. If $\tau$ is measure preserving and $\sigma$ commutes with $\tau$ then $\sigma$ is measure preserving.

Proof. Let $\mu(B)=m(\sigma(B)), B \in ß$. Therefore $\mu(\tau(B))=m(\sigma(\tau(B)))$ $=m(\tau(\sigma(B)))=m(\sigma(B))=\mu(B)$, i.e., $\mu$ is invariant under $\tau$. If $\tau(A)=A$ and $m(A)=0$ then $\sigma \in J$ implies $\mu(A)=0$. Similarly $\tau(A)=A$ and

Received by the editors June 20, 1966.

${ }^{1}$ Research support by the National Science Foundation, Grant GP-1816. 
$m(A)=1$ implies $\mu(A)=1$. It now follows by Theorem $1,[2]$ that $\mu(B)=m(B), B \in B$, i.e., $\sigma$ is measure preserving.

Corollary 1. If $\tau$ is mixing and $\sigma$ is a root of $\tau$ then $\sigma$ is mixing.

Proof. Let $\sigma^{p}=\tau$, hence $\sigma^{n}(A)=\tau^{[n / p]}\left(\sigma^{j}(A)\right)$, where $[i]$ denotes the largest integer such that $[i] \leqq i$, and $0 \leqq j \leqq p-1$. Given $\epsilon>0$ we may choose $k$ so large that $\left|m\left(\tau^{k}\left(\sigma^{j}(A)\right) \cap B\right)-m\left(\sigma^{j}(A)\right) m(B)\right|<\epsilon, 0 \leqq j$ $\leqq p-1$. However Theorem 1 implies $m\left(\sigma^{j}(A)\right)=m(A), 0 \leqq j \leqq p-1$. Therefore $n \geqq k p$ implies $\left|m\left(\sigma^{n}(A) \cap B\right)-m(A) m(B)\right|<\epsilon$.

In a similar manner we can prove

CoRollary 2. If $\tau$ is weakly mixing and $\sigma$ is a root of $\tau$ then $\sigma$ is weakly mixing.

Briefly, Corollaries 1 and 2 imply that in order to show a mixing (weakly mixing) transformation does not have a root it suffices to consider only mixing (weakly mixing) transformations. Since a transformation is weakly mixing if and only if it has continuous spectrum $[5$, p. 39] Corollary 2 says that if a transformation is a root of a transformation with continuous spectrum then it has continuous spectrum. In Theorem 4 below we obtain a similar result concerned with certain proper values of commuting transformations.

Definition. We say $\tau$ admits a $k$-stack with base $B$ if $\tau^{i}(B), 0 \leqq$ $i \leqq k-1$, are (essentially) disjoint and $m\left(\bigcup_{i=0}^{k-1} \tau^{i}(B)\right)=1$. In that case $\tau^{k}(B)=B$ a.e.

LEMMA 1. If $\tau$ admits a k-stack with base $B$ and $\sigma$ commutes with $\tau$ then $\sigma$ admits a $k$-stack with base $B$.

Proof. Let $B_{i}=\sigma(B) \cap \tau^{i}(B)$ and suppose $0<m\left(B_{i}\right)<m\left(\tau^{i}(B)\right)$ for some $i, 0 \leqq i \leqq k-1$. We then have $\tau\left(\bigcup_{j=0}^{k-1} \tau^{j}\left(B_{i}\right)\right)=\bigcup_{j=0}^{k-1} \tau^{j}\left(B_{i}\right)$ but $m\left(\bigcup_{j=0}^{k-1} \tau^{j}\left(B_{i}\right)\right)<1$. This contradicts the ergodicity of $\tau$. Hence either $\boldsymbol{m}\left(B_{i}\right)=0$ or $\sigma(B)=\tau^{i}(B)$ a.e. It follows that there exists a unique integer $i_{1}$ such that $\sigma(B)=\tau^{i_{1}}(B)$ a.e. Furthermore $i_{1} \neq 0$ for otherwise $\sigma(B)=B$ a.e. contradicting the ergodocity of $\sigma$. Now $\sigma^{2}(B)=\tau^{2 i_{1}(\bmod k)}(B)$ a.e. where $\sigma^{2}(B) \neq B$ if $k>2$ since $\sigma$ is ergodic. Proceeding inductively we find that $\sigma$ permutes the sets $\tau^{i}(B)$, $0 \leqq i \leqq k-1$, and finally $\sigma^{k}(B)=B$.

As a Corollary of Lemma 1 we have the following result [1, Lemma 5].

Lemma 2. If $\tau$ admits a $k$-stack then $\tau$ has no kth root.

Proof. If $\sigma^{k}=\tau$ then Lemma 1 implies $\sigma^{k}(B)=B$ contradicting $\boldsymbol{\sigma}^{k}(B)=\boldsymbol{\tau}(B)$. 
2. In this section we obtain ergodic measure preserving transformations with discrete spectrum satisfying (2) above. We shall utilize the following result which connects the geometric property of $\tau$ admitting a $k$-stack with the spectrum of $\tau$.

LEMMA 3. $\tau$ admits $a$-stack if and only if the kth roots of unity are proper values of $\tau$.

Proof. Let $r_{l}=e^{i 2 \pi l / k}, 0 \leqq l \leqq k-1$. Suppose $\tau$ admits a $k$-stack with base $B$. Let $f(x)=r_{l}, x \in \tau^{l}(B), 0 \leqq l \leqq k-1$. Therefore $f(\tau(x))=r_{1} f(x)$. In a similar manner we show $r_{2}, \cdots, r_{k-1}$ are proper values of $\tau$.

Conversely suppose $r_{1}$ is a proper value of $\tau$. Let $f(\tau(x))=r_{1} f(x)$ where $|f| \equiv 1$. Let $B=\{x: 0 \leqq \arg f(x)<2 \pi / k\}$. It suffices to assume $m(B)>0$. It is easily seen that $\tau^{i}(B), 0 \leqq i \leqq k-1$, are disjoint and $\bigcup_{i=0}^{k-1} \tau^{i}(B)$ is invariant under $\tau$. Thus $\tau$ admits a $k$-stack with base $B$.

LEMMA 4. If the kth roots of unity are proper values of $\tau$ then $\tau$ has no kth root.

Proof. By Lemma 3 and Lemma 2.

TheoreM 2. Let $k \geqq 2$ be prime. If $\tau$ has discrete spectrum then a necessary and sufficient condition that $\tau$ have a kth root is that the kth roots of unity be absent from its proper values.

Proof. If the $k$ th root of unity is a proper value of $\tau$, then according to Lemma $4, \tau$ has no $k$ th root. Conversely suppose the $k$ th root of unity is not a proper value of $\tau$. For the sake of completeness we sketch the proof, following Halmos [5]. Let $C^{*}$ be a maximal subgroup of the unit circle, which includes the proper values of $\tau$ but does not include the $k$ th root of unity. Then one shows in a straightforward fashion that the operation of raising to the $k$ th power is an automorphism of $C^{*}$. The inverse operation, say $u$, is a character of the set of proper values of $\tau$. Now $\tau$ is conjugate to the rotation on the character group defined by $S x=z x$, where $x$ is a character, and $z$ is defined by $z(c)=c$, for each proper value $c \in C^{*}$. Now it is easily verified that the rotation $u x$ is a $k$ th root of $S$, and thus $\tau$ has a $k$ th root.

THEOREM 3. There exists an ergodic measure preserving transformation with discrete spectrum satisfying (2) for arbitrary $P_{1}$ and $P_{2}$.

Proof. We may assume $P_{1}$ is not empty. Let $G_{1}$ be the smallest subgroup of the unit circle which contains the $p$ th roots of unity for $p \in P_{1}$. It follows that a $p$ th root of unity is not in $G_{1}$ if $p \in P_{2}$. By 
[5, Corollary 1, p. 48], there exists an ergodic measure preserving transformation $\tau$ with discrete spectrum $G_{1}$. That $\tau$ satisfies (2) now follows by Lemma 4 and Theorem 2 .

Concerning proper values of commuting transformations, we have the following result.

THEOREM 4. If the kth roots of unity are proper values of $\tau$, and $\sigma$ commutes with $\tau$ then the kth roots of unity are proper values of $\sigma$.

Proof. By Lemma 1 and Lemma 3.

In general ergodic transformations may commute and have disjoint sets of proper values. For example if $\tau(x)=x+\alpha(\bmod 1)$ and $\sigma(x)=x+\beta(\bmod 1)$ where $\alpha$ and $\beta$ are irrational and relatively prime.

3 . In this section we point out a class of invertible mixing transformations for which the existence of roots is easily seen. Wa first consider invertible transformations $T$ on the set of positive integers. Given $T$, we define a transformation $\tau_{T}$ on the unit interval utilizing the binary expansion. These transformations are introduced in [7]. Let $x=\sum_{i=1}^{\infty} b_{i}(x) 2^{-i}$ and define

$$
\tau_{T}(x)=\sum_{i=1}^{\infty} b_{T(i)}(x) 2^{-i}
$$

It follows at once that if $S^{p}=T$ then $\tau_{S}^{p}=\tau_{T}$. However it is not difficult to give examples where $\tau_{T}$ has a root although $T$ does not.

It is proven in [7] that $\tau_{T}$ is mixing if $T$ is antiperiodic, i.e., $T$ has no periodic points. A transparent example of an invertible mixing transformation with a square root may be obtained as follows. Let $T^{n}(1)=1+4 n, \quad n=1,2, \cdots, T^{-1}(1)=3, \quad$ and $T^{-n}(3)=3+4 n, n$ $=1,2, \cdots$. Let $T^{n}(2)=2+4 n, n=1,2, \cdots, T^{-1}(2)=4$, and $T^{-n}(4)=4+4 n, n=1,2, \ldots . T$ is now completely defined and decomposes into two disjoint antiperiodic orbits consisting of the even positive integers and the odd positive integers. A square root $S$ is uniquely determined by defining $S(1)=2$ and requiring $S^{2}(i)=T(i)$, $i=1,2, \cdots$. Therefore $\tau_{S}$ is a square root of $\tau_{T}$. The behavior of $\tau_{S}$ and $\tau_{T}$ is easily visualized by considering the images of binary intervals under $\tau_{T}$ and $\tau_{S}$. In order to construct invertible mixing transformations with roots of every order it is simply necessary to obtain $T$ with a countable number of antiperiodic orbits. A $p$ th root is obtained by grouping sets of $p$ orbits in an analogous manner as above.

If $T$ has only one antiperiodic orbit then $T$ cannot have a square root but it is not known if $\tau_{T}$ has a square root. This class of transformations may be considered as generalized 2-shifts $[5$, p. 8]. 


\section{REFERENCES}

1. J. R. Blum and N. Friedman, On roots of transformations, Z. Wahrscheinlichkeitstheorie und Verw. Gebiete 5 (1966), 1-5.

2. J. R. Blum and D. Hanson, On invariant probability measures. I, Pacific J. Math. 10 (1960), 1125-1129.

3. R. V. Chacon, Transformations having continuous spectrum (to appear).

4. - A geometric construction of measure preserving transformations. Proc. Fif th Berkeley Sympos. Math. Statist. and Prob. (to appear).

5. P. R. Halmos, Lectures on ergodic theory, Publ. Math. Soc. Japan No. 3, 1965.

6. - Square roots of measure preserving transformations, Amer. J. Math. 64 (1942), 153-166.

7. C. Standish, A class of measure preserving transformations, Pacific J. Math. 6 (1956), 553-564.

University of New Mexico 